\title{
Actitudes hacia la violencia, impulsividad, estilos parentales y conducta externalizada en adolescentes: comparación entre una muestra de población general y una muestra clínica
}

\author{
José Antonio Jiménez-Barbero ${ }^{1 *}$, José Antonio Ruiz-Hernández ${ }^{2}$, Antonio Pablo Velandrino-Nicolás ${ }^{3}$, Laura Llor-Zaragoza ${ }^{4}$ \\ 1 Universidad de Murcia. Grupo de Investigación de Psicología Social. Facultad de Psicología. Murcia (España). \\ 2 Universidad de Murcia. Departamento de Psiquiatria y Psicología Social. Facultad de Psicología. Murcia (España). \\ 3 Universidad de Murcia. Departamento de Psicología Básica y Metodología. Facultad de Psicología. Murcia (España). \\ 4 Universidad Católica San Antonio. Facultad de Ciencias Sociales y de la Comunicación. Departamento de Educación. Murcia (España).
}

\begin{abstract}
Resumen: Los Trastornos de Conducta Externalizantes (TCE) constituyen uno de los problemas de salud mental más comunes entre los adolescentes, y repercuten gravemente en el ámbito familiar, escolar y social. La finalidad de este estudio fue comparar adolescentes de población general sin diagnóstico de TCE con adolescentes de una muestra clínica en una serie de variables identificadas como predictoras de la de la conducta externalizada. El estudio incluyó a adolescentes con edades comprendidas entre 12-15 años de la Región de Murcia. Se empleó un diseño selectivo descriptivo y analítico, así como comparativo transversal en una muestra de 327 adolescentes, que se distribuyeron de la siguiente manera: (a) Grupo Clínico (GC), integrado por 59 participantes, y (b) Grupo General (GG), formado por 268 adolescentes.

Los resultados de este estudio indican que la conducta externalizada está relacionada principalmente con la impulsividad, aunque modulada por factores relacionados con la socialización familiar y cultural del adolescente. Asimismo, los adolescentes de la muestra clínica describen los estilos parentales que reciben como más autoritarios y menos inductivos que los adolescentes de población general.
\end{abstract}

Palabras clave: Trastornos de conducta; externalización; impulsividad; estilos parentales; actitudes.

\section{Introducción}

Los trastornos generalizados del desarrollo y los trastornos de conducta externalizantes (TCE), entre los que se incluyen el trastorno por déficit de atención e hiperactividad y el trastorno oposicionista-desafiante, están considerados como los diagnósticos más frecuentes entre la población infantil y adolescente (Gadow, Sprafkin \& Nolan, 2001). Los TCE, caracterizados por comportamientos manifiestos desajustados, agresividad, agitación psicomotora, desobediencia y comportamiento delincuente (Achenbach \& Edelbrock, 1984; Achenbach \& Ruffle, 2000), tienen gran repercusión en el ámbito escolar, donde se relacionan con episodios de violencia entre pares (Arseneault et al., 2006). Se ha informado que la exposición a este tipo de experiencias puede causar la aparición de: (a) problemas emocionales y psicosomáticos (Gini \& Pozzoli, 2009), (b) baja autoestima, depresión e ideación suicida (Evans, Hawton \& Rodham, 2004), o (c) comportamientos antisociales, como conductas delictivas o abuso de drogas, que a su vez pueden redundar en problemas legales, económicos y sociales (Cunningham \& Henggeler, 2001; Jiménez-Barbero, Ruiz-Hernández, LlorEsteban, \& Pérez-García, 2012). La evidencia indica que no existe un único factor causal de los TCE. Distintos estudios

* Dirección para correspondencia [Correspondence address]: José Antonio Jiménez-Barbero. Universidad de Murcia. Grupo de Investigación de Psicología Social. Facultad de Psicología. CP 30100 Espinardo, Murcia (Spain).Email: barbero49@hotmail.com
Title: Attitudes towards violence, impulsivity, parenting styles and externalized behavior in adolescents: comparison between a general and a clinical samples.

Abstract: Externalizing Behavior Disorders are one of the most common mental health problems among adolescents, and they have a severe impact on family, school, and social settings. The purpose of this study was to compare adolescents from the general population without an externalizing behavior diagnosis with adolescents from a clinical sample in a series of variables identified as predictors of externalizing behavior. The study included adolescents aged between 12-15 years from the Region of Murcia. A cross-sectional, descriptive, analytical design was used with a sample of 327 adolescents, who were distributed as follows: (a) Clinical Group, made up of 59 participants, and (b) General Group, made up of 268 adolescents.

The results of this study indicate that externalizing behavior is mainly related to Impulsivity, although modulated by factors related to the adolescent's family and cultural socialization. The adolescents of the clinical sample described the parental styles they received as more authoritarian and less in ductive than those of the adolescents from the general population.

Key words: Behavior disorders; externalization; impulsivity; parental styles; attitudes.

han señalado que las interacciones parentales, los factores ambientales y las propias características del niño, podrían estar influyendo en su desarrollo (Fraser \& Wray, 2008). Por otra parte, teorías como la Teoría Polivagal, explican que gran parte de la conducta social y de las emociones tienen importantes condicionantes fisiológicos (Porges, 1995). En este sentido, se ha propuesto que las deficiencias en la regulación emocional generadas dentro de las familias a través de procesos coercitivos amplifican la impulsividad favoreciendo el desarrollo de TCE en niños (Beauchaine, Gatzke-Kopp, \& Mead, 2007).

Precisamente la impulsividad ha sido identificada como un predictor fundamental de la conducta externalizada (Barratt, 1985; Olson, Schilling, \& Bates, 1999). Eysenck (1993) la definió como la tendencia a actuar de forma irreflexiva y sin considerar las consecuencias, relacionándolo con la existencia de déficits inhibitorios. Además de la impulsividad, se han encontrado evidencias de la importancia de las actitudes de los pares ante la violencia y hacia las víctimas de ésta en las conductas violentas en el ámbito escolar (Stevens, Van Oost, \& De Bourdeaudhuij, 2000), e incluso se ha postulado que la modificación de las actitudes de los jóvenes, podría reducir el riesgo de las mismas (Zun, Downey, \& Rosen, 2004). Desde este prisma, tanto la violencia, como la actitud hacia ella podrían ser factores de gran importancia en el desarrollo de conductas externalizantes antisociales (Hanish \& Guerra, 2002). Por otra parte, los estilos parentales también se han considerado relevantes para el desarrollo de estas conductas, llevándose a cabo diversos estudios con el fin 
de examinar el papel que tienen en la aparición de TCE en niños y adolescentes (Martínez-Ferrer, Murgui-Pérez, Musitu-Ochoa, \& Monreal-Gimeno, 2008). Un reciente estudio en estudiantes de Enseñanza Secundaria, encontró que tanto la impulsividad como los estilos educativos autoritarios o inconsistentes y las actitudes hacia la violencia como forma de potenciación de la autoestima o de manejar las relaciones sociales, se asociaban a la conducta externalizada en adolescentes (Jiménez-Barbero, Ruiz-Hernández, Llor-Esteban, \& Waschgler, 2014). Dichos resultados venían a confirmar los obtenidos por otros autores previamente (Dwairy, 2008; Dodge \& Crick, 1990; Martínez-Ferrer et al., 2008).

Sin embargo, la mayoría de estudios transversales sobre TCE en adolescentes, se han centrado en población general (Sprafkin, Volpe, Gadow, Nolan \& Kelly, 2002; Gadow, DeVincent, Pomeroy \& Azizian, 2004). Por este motivo, se planteó como objetivo principal del presente estudio, comparar adolescentes sin diagnóstico de TCE con adolescentes de una muestra clínica en una serie de variables identificadas en estudios previos como predictoras de la conducta externalizada (Jiménez-Barbero et al., 2014). También se planteó analizar las diferencias por edad, género, nivel cultural de los padres y convivencia familiar, entre ambos grupos.

\section{Método}

\section{Participantes}

El estudio incluyó a adolescentes con edades comprendidas entre 12-15 años de la Región de Murcia. Se obtuvo una muestra total de 327 adolescentes, de los cuales, 180 (55 $\%$ ) fueron varones y 147 (45\%), mujeres. La media de la edad fue de 13.5 (DT=.93). Siguiendo los criterios de control metodológico descritos en el apartado de procedimiento se eliminaron 16 casos al detectar problemas de sinceridad, por lo que la muestra final fue de 316.

Los participantes se distribuyeron de la siguiente manera: (a) Grupo Clínico (GC), integrado por 59 adolescentes $(18 \%)$ tratados de TCE según la clasificación de la CIE-10 (World Health Organization, 2010), y procedentes de Centros de Salud Mental Infanto-Juvenil de la Región de Murcia, seleccionados mediante un muestreo por conglomerados, de los cuales $42(71.2 \%)$, estaban diagnosticados de trastornos de conducta externalizantes; (b) Grupo General (GG), integrado por 268 adolescentes $(82 \%)$, que se obtuvo en un Instituto de Enseñanza Secundaria de la Región de Murcia, seleccionado por encontrarse situado en un barrio habitado por una población de características sociodemográficas similares a las del resto de la población urbana de la zona. Ambos grupos fueron reclutados durante el último trimestre de 2011 y primero de 2012 (Tabla 1).
Tabla 1. Descripción de variables sociodemográficas.

\begin{tabular}{|c|c|c|}
\hline \multirow[t]{2}{*}{$V A R I A B L E S$} & \multicolumn{2}{|c|}{ GRUPO } \\
\hline & GC & GG \\
\hline \multicolumn{3}{|l|}{ Edad } \\
\hline $12-13$ & $32(54.23 \%)$ & $121(45.1 \%)$ \\
\hline 14-15 & $27(45.77 \%)$ & $136(50.8 \%)$ \\
\hline \multicolumn{3}{|l|}{ Género } \\
\hline Niños & $41(69.5 \%)$ & $138(51.5 \%)$ \\
\hline Niñas & $18(30.5 \%)$ & $128(47.8 \%)$ \\
\hline \multicolumn{3}{|l|}{ Convivencia familiar } \\
\hline Con mi padre y con & $44(74.6 \%)$ & $224(83.6 \%)$ \\
\hline Mi madre & & \\
\hline Con mi madre & $11(18.6 \%)$ & $32(11.9 \%)$ \\
\hline Con mi padre & $2(3.4 \%)$ & $3(1.1 \%)$ \\
\hline No convivo con mis padres & $2(3.4 \%)$ & $3(1.1 \%)$ \\
\hline
\end{tabular}

\section{Instrumentos de evaluación}

El protocolo incluyó un cuestionario autoadministrado compuesto por 201 ítems, que recogió las variables sociodemográficas y las variables de estudio, y que requirió unos 35 minutos aproximadamente para su cumplimentación. El cuestionario que se empleó en el GC, incluyó además el diagnóstico principal.

Variables sociodemográficas. Se incluyó edad, género y convivencia familiar. La convivencia familiar se clasificó en 4 categorías: (1) con mi padre y con mi madre, (2) con mi madre, (3) con mi padre, o (4) no convivo con mis padres.

Externalización. Empleamos el cuestionario autoadministrado Youth Self-Report, de Achenbach (Achenbach et al., 1984; Achenbach et al., 2000), diseñado para obtener información directamente de los niños y adolescentes con edades comprendidas entre 11 y 18 años sobre diversas competencias y problemas de conducta. Consta de dos partes: la primera de ellas evalúa competencias deportivas, académicas y sociales, mientras que la segunda, compuesta por 112 ítems, evalúa conductas prosociales y conductas-problema. Para el presente estudio se utilizaron los ítems que medían los factores agresividad verbal y conducta delincuente, debido a su mayor consistencia interna, según estudios de validación en español de este instrumento (Lemos Giráldez, Vallejo Seco \& Sandoval Mena, 2002).

Impulsividad. La Escala de Impulsividad de Barratt, (BIS10) (Barratt, 1985), es uno de los instrumentos más utilizados para medir la impulsividad, presentando una alta consistencia interna, con niveles comprendidos entre .89 y .92 , por lo que se decidió aplicar en el presente estudio. En concreto, se empleó la subescala que mide la impulsividad motora, de la versión validada en español de dicho instrumento (Luengo, Carrillo de la Peña \& Otero, 1991).

Actitudes hacia la violencia. Se empleó el Cuestionario de Actitudes Hacia la Violencia de 25 ítems (CAHV-25) (RuizHernández, Llor, Puebla \& Llor-Esteban, 2009). Este instrumento consta de 4 factores: actitud hacia la violencia como forma de diversión ( 7 ítems, Cronbach=.78); actitud hacia la violencia para mejorar la autoestima (5 ítems, Cronbach=.78); actitud hacia la violencia para manejar los 
problemas y las relaciones sociales (habilidad social) (6 ítems, Cronbach $=.68)$; y actitud hacia la violencia percibida como defensa legítima ( 7 ítems, Cronbach=.72). El nivel de consistencia interna total del instrumento es alto (Cronbach=.90).

Estilos educativos parentales. Para el estudio de esta variable empleamos el Cuestionario sobre Estilos Disciplinarios de los Padres (Torrente-Hernández \& Vazsonyi, 2008). Es un instrumento validado y autoadministrado compuesto por 52 ítems, que clasifica los estilos educativos en inductivo, autoritario, permisivo y sobreprotector, basados en el modelo propuesto por Baumrind (1991). Los análisis de fiabilidad del cuestionario han dado como resultados un índice alfa de Cronbach $=.77$ para los estilos paternos $\mathrm{y}=.69$ para los maternos.

Sinceridad. Para controlar los problemas de sinceridad, se utilizaron dos procedimientos: por una parte, la subescala de sinceridad incluida en el CACIA (Cuestionario de AutoControl Infantil y Adolescente, Capafons \& Silva, 1998), que consideramos apropiada por su brevedad (14 ítems) y por su fácil comprensión y sencillez; y por otra parte una pregunta de sinceridad autoinformada.

\section{Procedimiento}

El presente estudio siguió una doble estrategia descriptiva y asociativa. Desde la perspectiva descriptiva, se puede definir como estudio selectivo transversal descriptivoanalítico, al perseguir entre sus objetivos el proporcionar información puntual sobre la población de estudio, así como la comparación de dos grupos mediante la exploración de las diferencias entre ambos en las variables de interés. Por otra parte, el análisis de datos, que incluyó la medición del grado de asociación entre dichas variables para cada grupo, permitió emplear una estrategia asociativa, por lo que el presente estudio emplea además un diseño comparativo transversal (Ato, López \& Benavente, 2013).

Para ello, se comparó una muestra de población general (GG), y una muestra clínica (GC). El GG se obtuvo en una única sesión, aprovechando el horario de clase del Instituto de Enseñanza Secundaria previamente seleccionado. Los adolescentes incluidos en el GC cumplimentaron el instrumento durante las correspondientes citas médicas, previa autorización de sus padres y aceptación de los responsables de los Centros. De la muestra final se excluyeron a aquellos participantes que no superaron el cuestionario de sinceridad. El proyecto fue aprobado por el Comité de Ética e Investigación Clínica del Hospital Universitario "Virgen de la Arrixaca”, y del Hospital Universitario "Morales Meseguer" (Murcia).

\section{Análisis de datos}

El análisis de datos se llevó a cabo usando el paquete estadístico SPSS (V.20.0.). Se realizaron pruebas Chi-Cuadrado de Pearson para comprobar si las puntuaciones en las variables independientes eran diferentes atendiendo a las variables sociodemográficas. Se estimaron las diferencias en función del sexo entre las variables predictoras, mediante análisis t de Student, calculándose los Tamaños del Efecto corespondientes (diferencia tipicada de medias, $\Delta$ de Cohen, Hedges \& Olkin, (1985)).

Con el fin de explorar las diferencias en las distintas variables entre el GG y el GC se llevó a cabo un análisis t de Student para muestras independientes, mediante comparación de medias. Posteriormente se analizó la relación entre las variables predictoras (impulsividad, estilos parentales, y actitudes hacia la violencia) y la variable criterio (externalización), mediante los coeficientes de correlación de Pearson.

\section{Resultados}

Tal y como nos muestra la Tabla 2, podemos observar diferencias en función del sexo en las variables Impulsividad, con valores superiores en mujeres ( $p=.047, \Delta=-.226$ ), estilos parentales maternos Inductivo $(p=.048, \Delta=-.234)$, con valores medios superiores entre mujeres, y Autoritario $(p=.045$, $\Delta=.242$ ) en los que se encuentran valores superiores entre varones. Respecto a los estilos parentales paternos, sólo se obtienen diferencias significativas en función del sexo en estilo Autoritario $(p=.024, \Delta=.274)$, que alcanza valores superiores en varones. Asimismo los resultados indican diferencias por sexo cercanas a la significación en Actitudes hacia la violencia como Defensa Legítima ( $p=.053, \Delta=.222)$. No obstante, teniendo en cuenta los tamaños de los efectos podemos asumir que las diferencias son bajas atendiendo a la clasificación de Cohen (1988).

En la Tabla 3 se recoge la comparación entre GG y GC mediante análisis t de Student. En este sentido, encontramos diferencias estadísticamente significativas en la variable Externalización, con valores superiores en el GC, observándose un tamaño del efecto considerable en los dos factores de la variable: Agresividad Verbal $(\Delta=-.516)$, y Conducta Delincuente $(\Delta=-.387, \mathrm{p}<.05)$. Asimismo, encontramos diferencias entre ambos grupos en las variables Impulsividad $(\Delta=$ $.384)$, estilo Autoritario Materno ( $\Delta=-.432)$ que presentan valores superiores entre el GC, y estilo Inductivo Materno y Paterno que, sin embargo, presentan valores superiores en el GG $(\Delta=.297, \mathrm{y} \Delta=.388$, respectivamente). 
Tabla 2. Análisis $t$ de Student mediante comparación de medias entre las variables predictoras, atendiendo al sexo.

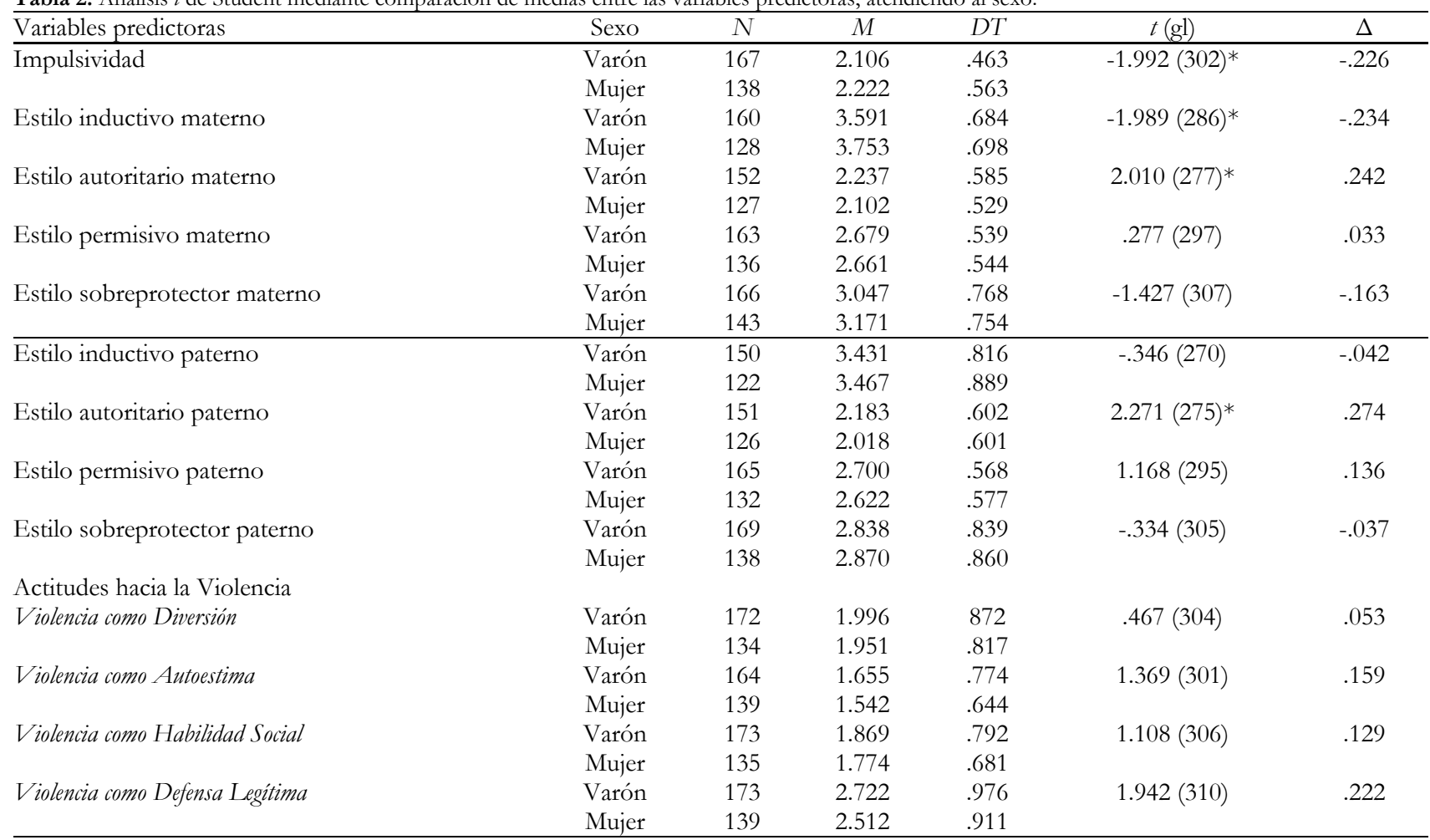

$* p<.05 ; D T=$ Desviación típica; $\mathrm{gl}=$ grados de libertad; $\Delta=$ tamaño del efecto.

Tabla 3. Análisis t de Student y tamaño del efecto para las distintas variables en el Grupo General (GG) y el Grupo Clínico (GC).

\begin{tabular}{|c|c|c|c|c|c|}
\hline \multirow[t]{2}{*}{ Variables } & \multicolumn{2}{|c|}{$M(D T)$} & \multirow[t]{2}{*}{$\mathrm{gl}$} & \multirow[t]{2}{*}{$t$} & \multirow[t]{2}{*}{$\Delta$} \\
\hline & GG & GC & & & \\
\hline \multicolumn{6}{|l|}{ Externalización } \\
\hline Agresividad verbal & $2.608(.920)$ & $2.981(1.096)$ & 300 & $2.366^{*}$ & -.516 \\
\hline Conducta delincuente & $1.583(.665)$ & $1.841(.699)$ & 301 & $2.318^{*}$ & -.378 \\
\hline \multicolumn{6}{|l|}{ Variables predictoras } \\
\hline Impulsividad & $2.142(.507)$ & $2.339(.517)$ & 288 & $2.321 *$ & -.384 \\
\hline Estilo inductivo materno & $3.718(.643)$ & $3.489(.900)$ & 271 & $-1.980^{*}$ & .297 \\
\hline Estilo autoritario materno & $2.139(.537)$ & $2.399(.666)$ & 263 & $2.756 * *$ & -.432 \\
\hline Estilo permisivo materno & $2.692(.547)$ & $2.636(.526)$ & 283 & -.621 & .104 \\
\hline Estilo sobreprotector materno & $3.107(.757)$ & $3.142(.894)$ & 293 & .275 & -.042 \\
\hline Estilo inductivo paterno & $3.531(.753)$ & $3.168(1.115)$ & 254 & $-2.616^{* *}$ & .388 \\
\hline Estilo autoritario paterno & $2.090(.555)$ & $2.206(.824)$ & 260 & 1.133 & -.168 \\
\hline Estilo permisivo paterno & $2.683(.537)$ & $2.710(.754)$ & 280 & .281 & -.041 \\
\hline Estilo sobreprotector paterno & $2.892(.807)$ & $2.682(1.158)$ & 291 & -1.468 & .213 \\
\hline \multicolumn{6}{|l|}{ Actitudes hacia la violencia } \\
\hline Como diversión & $2.045(.863)$ & $1.795(.746)$ & 289 & -1.763 & 0.301 \\
\hline Como autoestima & $1.639(.757)$ & $1.544(.506)$ & 286 & -.784 & 0.150 \\
\hline Como habilidad social & $1.871(.764)$ & $1.790(.648)$ & 291 & -.637 & 0.114 \\
\hline Como defensa legítima & $2.688(.963)$ & $2.513(.878)$ & 295 & -1.103 & 0.190 \\
\hline
\end{tabular}

${ }^{*} p<.05 ; * * p<.001 ; \mathrm{GG}=$ Grupo General; GC=Grupo Clínico; $D T=$ Desviación típica; gl= grados de libertad; $\Delta=$ tamaño del efecto.

En lo relativo a la Agresividad Verbal, mediante el análisis de correlación realizado (Tabla 4) observamos en el GG una fuerte correlación con la Impulsividad $(r=.625, p<.01)$, y moderada con estilo Autoritario Materno, $(r=.482, p<.01)$. También correlacionó con los estilos maternos Permisivo y Sobreprotector, aunque en estos casos la correlación era débil. Respecto a los estilos paternos, correlacionó moderada- mente con el Autoritario $(r=.343, p<.01)$, mientras que las correlaciones con el Permisivo y Sobreprotector fueron débiles. Además se observó una correlación fuerte con todos los factores de la variable Actitudes hacia la Violencia.

Por otra parte, Conducta Delincuente presentó una correlación fuerte con Impulsividad $(\mathrm{r}=.575, \mathrm{p}<.01)$, y con el estilo Autoritario Materno $(r=.583, p<.01)$, y moderada con 
el estilo Permisivo Materno $(r=.273, p<.01)$, Autoritario Paterno $(r=.359, p<.01)$, y Permisivo Paterno $(r=.286, p<.01)$. También encontramos correlaciones significativas inversas aunque moderadas, con los estilos Inductivos Materno $(r=-$ $.226, p<.01)$, y Paterno $(r=-.187, p<.01)$. Asimismo, al igual que ocurría con Agresividad Verbal, presenta una correlación fuerte con todos los factores de la variable actitudes hacia la violencia.

En el caso del GC (Tabla 4), se obtienen resultados similares, observándose también fuerte correlación entre
Agresividad Verbal e Impulsividad $(r=.625, p<.01)$. Respecto a los estilos parentales, esta variable presentó una correlación moderada con los estilos autoritarios materno y paterno (respectivamente, .425, y .324, $p<.01$ ). Por otro lado, la variable Conducta Delincuente presentó una correlación fuerte y directa con Impulsividad $(r=.512, p<.01)$, y moderada e inversa con estilo Inductivo Paterno $(r=-.364, p<.05)$. Con los factores de la variable actitudes hacia la violencia, correlaciona de forma moderada y directa en todos los casos.

Tabla 4. Correlaciones de Pearson entre la variable "Externalización” y las variables predictoras en el Grupo General y Grupo Clínico.

\begin{tabular}{|c|c|c|c|c|}
\hline \multirow[t]{3}{*}{ Variables Predictoras } & \multicolumn{4}{|c|}{ Externalización } \\
\hline & \multicolumn{2}{|c|}{ GG } & \multicolumn{2}{|c|}{ GC } \\
\hline & Agresividad Verbal & Conducta Delincuente & Agresividad Verbal & Conducta Delincuente \\
\hline Impulsividad & $.625^{* *}$ & $.575^{* *}$ & $.625^{* *}$ & $.512^{* *}$ \\
\hline \multicolumn{5}{|l|}{ Estilos parentales } \\
\hline Inductivo materno & -.113 & $-.226^{* *}$ & -.098 & -.273 \\
\hline Autoritario materno & $.482 * *$ & $.583^{* *}$ & $.405^{* *}$ & .224 \\
\hline Permisivo materno & $.199 * *$ & $.273^{* *}$ & .072 & -.035 \\
\hline Sobreprotector materno & $.213^{* *}$ & -.044 & .179 & -.140 \\
\hline Inductivo paterno & -.119 & $-.187 * *$ & -.182 & $-.364 *$ \\
\hline Autoritario paterno & $.343^{* *}$ & $.359 * *$ & $.324^{*}$ & .235 \\
\hline Permisivo paterno & $.165^{*}$ & $.283^{* *}$ & .077 & .019 \\
\hline Sobreprotector paterno & $.139 *$ & .092 & .089 & -.275 \\
\hline \multicolumn{5}{|l|}{ Actitudes hacia la violencia } \\
\hline Violencia como Diversión & $.497 * *$ & $.601 * *$ & .163 & $.468 * *$ \\
\hline Violencia como Autoestima & $.460^{* *}$ & $.573^{* *}$ & .233 & $.421 * *$ \\
\hline Violencia como Habilidad social & $.489^{* *}$ & $.583^{* *}$ & .178 & $.595^{* *}$ \\
\hline Violencia como Defensa legítima & $.485^{* *}$ & $.555^{* *}$ & $.339 * *$ & $.342 * *$ \\
\hline
\end{tabular}

${ }^{*} p<.05$ (bilateral); ${ }^{* *} p<.01$ (bilateral); GG= Grupo General; GC=Grupo Clínico.

\section{Discusión}

Nuestro estudio sólo encontró diferencias significativas en algunas de las variables predictoras de los TCE en función del sexo, lo cual está en la línea de resultados obtenidos por trabajos recientes (Rosa-Alcázar, Parada-Navas \& RosaAlcázar, 2014). Las diferencias, en este caso, se observaron en Impulsividad, con valores superiores entre las mujeres, contradiciendo los resultados obtenidos en las últimas décadas (Eysenck, 1967; Barratt \& Patton, 1983), aunque coincide con trabajos más recientes (Jiménez-Barbero, RuizHernández, Llor-Esteban, Llor-Zaragoza, \& Pérez García, 2013; Jiménez-Barbero et al., 2014). También se obtuvieron diferencias respecto al estilo parental autoritario, tanto paterno como materno, con valores superiores en varones en ambos casos. En este sentido, se ha apuntado que los niños varones están expuestos con frecuencia a disciplina familiar más dura que las niñas (es decir, están sometidos a mayor régimen de coerción física y verbal), lo cual podría estar asociado a una mayor incidencia en éstos de TCE (Meier, Slutske, Heath \& Martin, 2009). Además esta hipótesis permitiría explicar, al menos en parte, la mayor tendencia de las niñas a desarrollar trastornos de índole internalizador (Messer, Goodman, Rowe, Meltzer \& Maughan, 2006; Van Roy, Grøholt, Heyerdahl \& Clench-Aas, 2006).

Como era de esperar, los resultados de este estudio confirman que el nivel de externalización es superior entre ado- lescentes diagnosticados de TCE, que entre adolescentes no clínicos. Es destacable el hecho de que los adolescentes con TCE presentan mayor nivel de impulsividad que los del GG, lo cual parece indicar que entre las causas de estos trastornos, ejercen un papel muy relevante los condicionantes psicofisiológicos (Porges, 1995), en especial la impulsividad (Olson et al., 1999; Eysenck H.J., \& Eysenck M.W., 1985). Simultáneamente, los adolescentes de la muestra clínica informaron de estilos parentales más autoritarios y menos inductivos que los de población general. Estos resultados son consistentes con las hipótesis que presentamos en un estudio previo (Jiménez-Barbero et al., 2014), así como con resultados obtenidos por otros autores (Barratt, 1994; Mitchell et al., 2009; Olson et al., 1999), en los que se destaca la influencia de estilos autoritarios en el desarrollo de conductas externalizadas. Además, el estilo parental inductivo, que se caracteriza por la utilización del razonamiento, favoreciendo el locus de control interno sobre las propias acciones y la internalización de valores y normas (Krevans \& Gibbs, 1996), presenta niveles superiores en el GG, lo cual coincide con estudios que afirman que el empleo de modelos inductivos de socialización familiar podría estar contribuyendo a reducir la aparición de este tipo de trastornos en adolescentes (Eisenberg et al., 2001; Jiménez-Barbero et al., 2014).

Por otra parte, el análisis de correlaciones muestra que la asociación entre impulsividad y externalización es fuerte en ambos grupos, lo que nos confirma la importancia en general de esta variable en la aparición de conductas externaliza- 
das (Peach \& Gaultney, 2013). Asimismo, encontramos que, aunque en el GG, la mayor parte de las variables predictoras correlacionan con las dos dimensiones de externalización (agresividad verbal y conducta delincuente), no ocurre así en el GC, donde los análisis se limitan a mostrar una fuerte asociación de externalización con estilos parentales autoritarios. Es decir, los resultados parecen indicar que los estilos parentales, con la salvedad del estilo autoritario respecto a la agresividad verbal, no estarían influyendo en la conducta externalizada de los adolescentes con TCE. Esta aparente contradicción podría deberse al hecho de que el GC está constituido por adolescentes que ya están recibiendo tratamiento con terapias familiares y psicofarmacológicas, lo que estaría modulando la influencia de una socialización familiar adversa (Edwards, Céilleachair, Bywater, Hughes \& Hutchings, 2007).

Por otra parte, se observa, especialmente en el GG, una correlación fuerte entre externalización y actitudes hacia la violencia, lo que podría ilustrar la relación entre conducta y actitud, una cuestión muy debatida en Psicología Social. En este contexto, se ha trabajado desde el supuesto de que el conocimiento de la actitud de una persona, servirá para conocer, cuando menos, el marco general de su actuación (Fazio, 1989; Fazio, 2007). Se ha informado que las actitudes influyen sobre la conducta de manera más directa y automática en situaciones en las que se debe actuar rápidamente, y que la relación entre conducta y actitud es máxima cuando los sujetos no utilizan procesos deliberativos a la hora de llevar a cabo sus comportamientos (Fazio, Roskos-Ewoldsen \& Powell, 1994), es decir en las conductas impulsivas. Dicho de otro modo, la impulsividad, que como hemos visto, tiene una gran influencia tanto en la conducta externalizada en adolescentes, como en el desarrollo de TCE, estaría facili-

\section{Referencias}

Achenbach, T. M. \& Edelbrock, C. S. (1984). Psychopathology of childhood. Annual Review of Psychology, 35, 227-256.

Achenbach, T. M. \& Ruffle, T. M. (2000). The Child Behavior Checklist and related forms for assessing behavioral/emotional problems and competencies. Pediatrics in Review, 21, 265-271.

Arseneault, L., Walsh, E., Trzesniewski, K., Newcombe, R., Caspi, A., \& Moffitt, T. E. (2006). Bullying victimization uniquely contributes to adjustment problems in young children: a nationally representative cohort study. Pediatrics, 118, 130-138. doi:10.1542/peds.2005-2388

Ato, M., López, J. J., \& Benavente, A. (2013). Un sistema de clasificación de los diseños de investigación en psicología. Anales de Psicología, 29, 10381059. doi: 10.6018/analesps.29.3.178511

Barratt, E. (1985). Impulsiveness subtraits: Arousal and information processing. In Spence J. \& Izard (Eds.), Motivation, emotion and personality (pp. 137-146). Amsterdam: Elsevier.

Barratt, E. S. (1994). Impulsiveness and Aggression. In Monahan J. \& Steadman H.J. (Eds.), Violence and Mental Disorder (pp. 61-79). Chicago: University of Chicago Press.

Barratt, E. S. \& Patton, J. H. (1983). Impulsivity: cognitive, behavioural and psychophysiological correlates. In Zuckerman M. (Ed.), Biological bases of sensation seeking, impulsivity, and anxiety. (pp. 77-122). Hillsdale, New Jersey: Lawrence Erlbaum Associates.

Beauchaine, T. P., Gatzke-Kopp, L., \& Mead, H. K. (2007). Polyvagal Theory and Developmental Psychopathology: Emotion Dysregulation and tando que actitudes favorables hacia la violencia se traduzcan en conductas violentas.

En su conjunto, los resultados de nuestro estudio, que confirman en líneas generales los obtenidos por trabajos similares, parecen indicar que la conducta externalizada está relacionada principalmente con la impulsividad, aunque modulada por factores relacionados con la socialización familiar y cultural del adolescente. Sólo en aquellos casos en los que coinciden niveles elevados de impulsividad con estilos parentales adversos, basados en la coerción y la imposición (autoritarios), y no en la aceptación e implicación (inductivos/autoritativos), estos adolescentes presentan alto riesgo de desarrollar TCE.

Este estudio, sin embargo, presenta algunas limitaciones, que deben ser consideradas. La muestra general (GG), no fue obtenida mediante un muestreo probabilístico, lo que podría afectar a la generalización de los resultados. Por otra parte, aunque esto sí se hizo para el reclutamiento de la muestra clínica, el tamaño de ésta fue reducido, lo que impidió la realización de análisis más complejos, que hubieran permitido la elaboración de modelos predictivos de los TCE. Otra limitación de este trabajo, fue que se emplearon únicamente cuestionarios autorreportados, por lo que no se contempla la perspectiva de padres o profesores sobre las variables estudiadas.

Aunque hay límites en nuestro estudio, consideramos que los resultados proporcionan información relevante sobre la etiología de los TCE. Las limitaciones mencionadas recomiendan el desarrollo de futuras investigaciones que permitan analizar elementos personales y contextuales para entender más y mejor el inicio y mantenimiento de los trastornos de conducta externalizante en adolescentes.

Conduct Problems from Preschool to Adolescence. Biological Psychology, 74, 174-184.

Cunningham, P. B. \& Henggeler, S. W. (2001). Implementation of an Empirically Based Drug and Violence Prevention and Intervention Program in Public School Settings. Journal of Clinical Child Psychology, 30, 221232. doi:10.1207/S15374424JCCP3002_9

Dodge, K. A. \& Crick, N. R. (1990). Social information-processing bases of aggressive behavior in children. Personality and Social Psychology Bulletin, 16, 8-22.

Dwairy, M. A. (2008). Parental Inconsistency Versus Parental Authoritarianism: Associations with Symptoms of Psychological Disorders. Journal of Youth and Adolescence, 37, 616-626. doi:10.1007/s10964-007-9169-3

Edwards, R. T., Céilleachair, A., Bywater, T., Hughes, D. A., \& Hutchings, J. (2007). Parenting programme for parents of children at risk of developing conduct disorder: cost effectiveness analysis. BMJ, 334, 682. doi:http://dx.doi.org/10.1136/bmj.39126.699421.55

Eisenberg, N., Cumberland, A., Spinrad, T. L., Fabes, R. A., Shepard, S. A., Reiser, M. et al. (2001). The Relations of Regulation and Emotionality to Children's Externalizing and Internalizing Problem Behavior. Child Development, 72, 1112-1134.

Evans, E., Hawton, K., \& Rodham, K. (2004). Factors associated with suicidal phenomena in adolescents: a systematic review of populationbased studies. Clinical Psychology Review, 24, 957-979. doi:10.1016/j.cpr.2004.04.005 
Eysenck H.J. (1967). The biological basis of personality. Sprinfgield: Transaction Pub.

Eysenck H.J. \& Eysenck M.W. (1985). Personality and individual differences: a natural science approach. New York: Plenum Press.

Fazio, R. H. (1989). On the power and functionality of attitudes: the role of attitude accessibility. In Pratkanis A.R., Breckler S.J., \& Greenwald A.G. (Eds.), Attitude Structure and Function (pp. 153-179). Erlbaum: Hillsdale L.

Fazio, R. H. (2007). Attitudes as Object-Evaluation Associations of Varying Strength. Social Cognition, 25, 603-637. doi:10.1521/soco.2007.25.5.603

Fazio, R. H., Roskos-Ewoldsen, D. R., \& Powell, M. C. (1994). Attitudes, perception, and attention. In P.M.Niedenthal \& S. Kitayama (Eds.), The heart's eye: Emotional influences in perception and attention (pp. 197-216). San Diego, CA, US: Academic Press.

Fraser, A. \& Wray, J. (2008). Oppositional Defiant Disorder. Australian Family Psysician, 37, 402-405.

Gadow, K. D., DeVincent, C. J., Pomeroy, J., \& Azizian, A. (2004). Psychiatric Symptoms in Preschool Children with PDD and Clinic and Comparison Samples. Journal of Autism and Developmental Disorders, 34, 379393. doi:10.1023/B:JADD.0000037415.21458.93

Gadow, K. D., Sprafkin, J., \& Nolan, E. E. (2001). DSM-IV symptoms in community and clinic preschool children. Journal of the American Academy of Child and Adolescent Psychiatry., 40, 1383-1392. doi:10.1097/00004583200112000-00008

Gini, G. \& Pozzoli, T. (2009). Association between bullying and psychosomatic problems: a meta-analysis. Pediatrics, 123, 1059-1065. doi:10.1542/peds.2008-1215

Hanish, L. D. \& Guerra, N. G. (2002). A longitudinal analysis of patterns of adjustment following peer victimization. . Development and Psychopathology, 14, 69-89. doi:http://dx.doi.org/

Hedges, L. \& Olkin, I. O. (1985). Statistical Methods for Meta-Analysis. Orlando: Academic Press.

Jiménez-Barbero, J. A., Ruiz-Hernández, J. A., Llor-Esteban, B., LlorZaragoza, L., \& Pérez García, M. (2013). Efficacy of a brief intervention on attitudes to reduce school violence: A randomized clinical trial. Chil dren and Youth Services Review, 35, 1313-1318. doi:http://dx.doi.org/10.1016/i.childyouth.2013.05.010

Jiménez-Barbero, J. A., Ruiz-Hernández, J. A., Llor-Esteban, B., \& PérezGarcía, M. (2012). Effectiveness of antibullying school programmes: A systematic review by evidence levels. Children and Youth Services Review, 34, 1646-1658. doi:10.1016/j.childyouth.2012.04.025

Jiménez-Barbero, J. A., Ruiz-Hernández, J. A., Llor-Esteban, B., \& Waschgler, K. (2014). Influence of attitudes, impulsivity, and parental styles in adolescents' externalizing behavior. Journal of Health Psychology. doi:10.1177/1359105314523303

Krevans, J. \& Gibbs, J. C. (1996). Parents' Use of Inductive Discipline: Relations to Children's Empathy and Prosocial Behavior. Child Development, 67, 2605-3400. doi:10.1111/j.1467-8624.1996.tb01913.x

Lemos Giráldez, S., Vallejo Seco, G., \& Sandoval Mena, M. (2002). Estructura factorial del Youth Self-Report (YSR). Psicothema, 14, 816-822.

Luengo M.A., Carrillo de la Peña M.T., \& Otero J.M. (1991). The components of impulsiveness: a comparison of the 1.7 Impulsiveness Questionnaire and the Barratt Impulsiveness Scale. Personality and Individual Differences, 12, 657-667.
Martínez-Ferrer, B., Murgui-Pérez, S., Musitu-Ochoa, G., \& MonrealGimeno, M. C. (2008). El rol del apoyo parental, las actitudes hacia la escuela y la autoestima en la violencia escolar en adolescentes. International Journal of Clinical and Health Psychology, 8, 679-692.

Meier, M. H., Slutske, W. S., Heath, A. C., \& Martin, N. G. (2009). The role of harsh discipline in explaining sex differences in conduct disorder: A study of opposite-sex twin pairs. Journal of Abnormal Child Psychology, 37, 653-664.

Messer J., Goodman R., Rowe R., Meltzer H., \& Maughan B. (2006). Preadolescent conduct problems in girls and boys. J.Am.Acad.Child Adolesc.Psychiatry, 45, 184-191.

Mitchell, S. J., Lewin, A., Horn, I. B., Rasmussen, A., Sanders-Phillips, K., Valentine, D. et al. (2009). Violence Exposure and the Association between Young African American Mothers' Discipline and Child Problem Behavior. Academic Pediatrics, 9, 157-163. doi:10.1016/j.acap.2009.02.003.

Olson, S. L., Schilling, E. M., \& Bates, J. E. (1999). Measurement of Impulsivity: Construct Coherence, Longitudinal Stability, and Relationship with Externalizing Problems in Middle Childhood and Adolescence. Journal of Abnormal Child Psychology, 27, 151-165. doi:10.1023/A:1021915615677

Peach, H. D. \& Gaultney, J. F. (2013). Sleep, Impulse Control, and Sensation-Seeking Predict Delinquent Behavior in Adolescents, Emerging Adults, and Adults. Journal Of Adolescent Health, 53, 293-299. doi:http://dx.doi.org/10.1016/i.jadohealth.2013.03.012

Porges, S. W. (1995). Orienting in a defensive world: mammalian modifications of our evolutionary heritage. A Polyvagal Theory. Psychophysiology, 32, 301-318

Rosa-Alcázar, A. I., Parada-Navas, J. L., \& Rosa-Alcázar, A. (2014). Síntomas psicopatológicos en adolescentes españoles: relación con los estilos parentales percibidos y la autoestima. Anales de Psicología, 30, 133-142. doi:http://dx.doi.org/10.6018/analesps.30.1.165371

Ruiz-Hernández, J. A., Llor, L., Puebla, T., \& Llor-Esteban, B. (2009). Evaluación de las creencias actitudinales hacia la violencia en centros educativos: el CAHV-25. European Journal of Education and Psychology, 2, 25-35.

Sprafkin, J., Volpe, R. J., Gadow, K. D., Nolan, E. E., \& Kelly, K. (2002). A DSM-IV-referenced screening instrument for preschool children: The Early Childhood Inventory-4. Journal of the American Academy of Child and AdolescentPsychiatry, 41, 604-612.

Stevens, V., Van Oost, P., \& De Bourdeaudhuij, I. (2000). The effects of an anti-bullying intervention programme on peers' attitudes and behaviour. Journal of Adolescence, 23, 21-34. doi:10.1006/jado.1999.0296

Torrente-Hernández, G. \& Vazsonyi, A. T. (2008). The salience of the family in antisocial and delinquent behaviors among spanish adolescents. The Journal of Genetic Psychology, 169, 189-197.

Van Roy, B., Grøholt, B., Heyerdahl, S., \& Clench-Aas, J. (2006). Selfreported strengths and difficulties in a large Norwegian population 1019 years: Age and gender specific results of the extended SDQquestionnaire. European Child \& Adolescent Psychiatry, 15, 189-198.

World Health Organization (2010). International Statistical Classification of Diseases and Related Health Problems 10th Revision. Geneva: WHO.

Zun, L. S., Downey, L., \& Rosen, J. (2004). An emergency departmentbased program to change attitudes of youth toward violence. The Journal of Emergency Medicine, 26, 247-251. doi:10.1016/j.jemermed.2003.06.008

(Articulo recibido: 21-03-2014; revisado: 16-02-2015; aceptado: 08-03-2015) 